\title{
El employer branding en las organizaciones que internacionalizan servicios de operación logística en Bogotá Colombia
}

\section{Employer branding in organizations that internationalize logistics operation services in Bogota Colombia}

\author{
Campo Elías López-Rodríguez*1, Samir Ricardo Neme-Chaves² \\ ${ }^{1}$ Corporación Universitaria Minuto de Dios - UNIMINUTO, Colombia \\ ${ }^{2}$ Universidad Santo Tomás, Colombia
}

Recibido el 12 de septiembre de 2019; aceptado el 2 de junio de 2020

Disponible en Internet el: 11 de junio de 2020

\section{Resumen}

El employer branding hace referencia a la gestión de marca como una alternativa para aumentar el compromiso y satisfacción de los empleados con la organización. El objetivo de la presente investigación es identificar la percepción que poseen los colaboradores de las organizaciones que internacionalizan servicios de operación logística en la ciudad de Bogotá, Colombia respecto a los diversos factores que sustentan las dimensiones del employer branding. Para ello, se utilizó el coeficiente de correlación de Pearson, el análisis de correspondencias simples y la normalización simétrica en las diferentes asociaciones obtenidas, las cuales muestran que las empresas participantes se han centrado en el hecho de capacitar a los empleados de nivel operativo, pero han descuidado a los empleados de alta y media gerencia; los empleados más jóvenes consideran que están bien remunerados, no así los mayores. Finalmente, las mujeres consideran que tienen límites respecto las labores que pueden llevar a cabo en dichas empresas, a diferencia de los hombres.

Código JEL: C46, E24, M10, M12, M54

Palabras clave: Internacionalización de servicios; Operación logística; Cliente interno; Fidelización del empleado

\footnotetext{
*Autor para correspondencia.

Correo electrónico: clopezr3@ uniminuto.edu.co (C. E. López-Rodríguez).

La revisión por pares es responsabilidad de la Universidad Nacional Autónoma de México. 


\begin{abstract}
The employer branding refers to brand management as an alternative to increase employee commitment and satisfaction with the organization. The objective of this research is to identify the perception that collaborators of organizations that internationalize logistics operation services in the city of Bogotá, Colombia have regarding the various factors that support the employer branding dimensions. To do this, Pearson's correlation coefficient, simple correspondence analysis and symmetric normalization in the different associations obtained were used, which show that the participating companies have focused on training employees at the operational level, but they have neglected high and middle management employees; Younger employees believe they are well paid, not older ones. Finally, women consider that they have limits on the work they can carry out in these companies, unlike men.
\end{abstract}

JEL Code: C46, E24, M10, M12, M54

Keywords: Internationalization of services; Logistics operation; Internal customer; Employee loyalty

\title{
Introduction
}

La importancia del employer branding en las organizaciones del siglo XXI radica en el protagonismo de sus empleados como uno de los principales stakeholders a los que la empresa debe otorgar una propuesta de valor que cumpla con sus expectativas como cliente interno, tal como lo manifiestan Kotler y Keller (2012) cuando desde su concepto de marketing holístico resaltan el marketing interno como una actividad estratégica de las compañías; y más aún cuando el desarrollo de la nueva economía se fundamenta en el conocimiento y en el capital humano (Villegas, Hernández y Salazar, 2017); de esta manera elementos como la remuner0ación justa, el buen ambiente laboral, la seguridad, la identificación con la empresa y un buen servicio de salud, entre otros hacen parte de aquellos atributos que esperan encontrar los empleados en su lugar de trabajo (Sempértegui y Bravo, 2018),

La necesidad de las organizaciones en incrementar su productividad ubica a los colaboradores en un escenario relevante para tal fin, siendo evidente que los profesionales con talento generan valor para las organizaciones donde están vinculados, pero igualmente desarrollan altas expectativas que las empresas deben cumplir (Fernández-Lores, Avelló, Gavilán y Blasco, 2014). La capacidad de los directivos de satisfacer a sus empleados garantiza que los procesos administrativos, no sólo en el área de talento humano sino en las demás áreas de gestión marchen de acuerdo a las dinámicas propias del mercado y del entorno competitivo (Hadi y Ahmed, 2018), garantizando la optimización de estrategias ante los cambios que surgen tanto en el micro como en el macroambiente organizacional (Cardona-Arbeláez, Henríquez-Fuentes, Rada-Llanos, Martínez y Bonnet, 2017). 
El talento humano visualiza el conjunto competencias que tienen los colaboradores; sin embargo, las débiles estrategias de endomarketing, el cual actúa como un ente facilitador de la relación entre el producto, el servicio, la marca y los trabajadores (Jiménezy Guardia, 2017), serán los principales obstáculos para potencializar el capital humano, en el cual todas las competencias del personal aportan significativamente a la productividad de las empresas, y más aún, cuando éstas tienen una incidencia global como lo son las organizaciones que internacionalizan servicios de operación logística. Por ello, Bilińska-Reformat y Stańczyk (2018) sostienen que la gestión del marketing interno requiere de una visión holística para convertirlo en una herramienta positiva para el negocio y su reputación frente a los stakeholders.

La importancia del employer branding en las organizaciones del siglo XXI radica en el protagonismo de sus empleados como uno de los principales stakeholders a los que la empresa debe otorgar una propuesta de valor que cumpla con sus expectativas como cliente interno, tal como lo manifiestan Kotler y Keller (2012) cuando desde su concepto de marketing holístico resaltan el marketing interno como una actividad estratégica de las compañías; y más aún cuando el desarrollo de la nueva economía se fundamenta en el conocimiento y en el capital humano (Villegas, Hernández y Salazar, 2017); de esta manera elementos como la remuner0ación justa, el buen ambiente laboral, la seguridad, la identificación con la empresa y un buen servicio de salud, entre otros hacen parte de aquellos atributos que esperan encontrar los empleados en su lugar de trabajo (Sempértegui y Bravo, 2018),

La necesidad de las organizaciones en incrementar su productividad ubica a los colaboradores en un escenario relevante para tal fin, siendo evidente que los profesionales con talento generan valor para las organizaciones donde están vinculados, pero igualmente desarrollan altas expectativas que las empresas deben cumplir (Fernández-Lores, Avelló, Gavilán y Blasco, 2014). La capacidad de los directivos de satisfacer a sus empleados garantiza que los procesos administrativos, no sólo en el área de talento humano sino en las demás áreas de gestión marchen de acuerdo a las dinámicas propias del mercado y del entorno competitivo (Hadi y Ahmed, 2018), garantizando la optimización de estrategias ante los cambios que surgen tanto en el micro como en el macroambiente organizacional (Cardona-Arbeláez, Henríquez-Fuentes, Rada-Llanos, Martínez y Bonnet, 2017).

El talento humano visualiza el conjunto competencias que tienen los colaboradores; sin embargo, las débiles estrategias de endomarketing, el cual actúa como un ente facilitador de la relación entre el producto, el servicio, la marca y los trabajadores (Jiménez y Guardia, 2017), serán los principales obstáculos para potencializar el capital humano, en el cual todas las competencias del personal aportan significativamente a la productividad de las empresas, y más aún, cuando éstas tienen una incidencia global como lo son las organizaciones que internacionalizan servicios de operación logística. Por ello, Bilińska-Reformat y Stańczyk 
(2018) sostienen que la gestión del marketing interno requiere de una visión holística para convertirlo en una herramienta positiva para el negocio y su reputación frente a los stakeholders.

En el escenario colombiano, los operadores logísticos son una modalidad de tercerización reciente, la cual viene posicionándose en el mercado gracias a la eficiencia de las operaciones logísticas, la creciente presencia de economías de escala, la simplificación de las operaciones en la cadena de abastecimiento y la decisión de cambio de costos fijos por variables (González Correa, 2015); no obstante, debido a esta realidad tanto los usuarios como los prestadores de servicios logísticos se han venido enfrentando a diversas barreras que impactan hoy el desempeño logístico en el país en términos de infraestructura, gestión de aduanas y gestión empresarial. Un estudio del Departamento Nacional de Planeación-DPN (2016) resalta de las anteriores barreras para los usuarios de servicios logísticos los altos costos de transporte, las insuficientes carreteras, puertos y aeropuertos, y la falta de sistemas de información logística; en el caso de los prestadores de servicios logísticos las barreras más relevantes son la falta de zonas adecuadas para carga y descarga, la deficiente infraestructura vial y la falta de talento humano en logística, siendo este último el elemento que se relaciona con las realidades del employer branding en esta actividad económica.

Desde lo anterior, se pretende identificar la percepción que poseen los colaboradores de las organizaciones que internacionalizan servicios de operación logística en la ciudad de Bogotá, Colombia respecto a los diversos factores de las dimensiones del employer branding persiguiendo mejorar el ambiente laboral dentro de estas a través fidelización del cliente interno el cual posee un alto protagonismo en el desarrollo de las actividades estratégicas, tácticas y operativas de dichas empresas, teniendo en cuenta lo propuesto por Vega y Serna (2017) cuando plantean que este concepto se originó como producto de la investigación en el sector de servicios, al reconocer la necesidad de los gerentes de enfocarse internamente tanto en los empleados como en los clientes.

Desde esta forma, se propone la siguiente formulación del problema: ¿Cuáles son las asociaciones existentes entre las dimensiones del employer branding y los factores sociodemográficos de los clientes internos en las organizaciones que internacionalizan servicios de operación logística en la ciudad de Bogotá, Colombia? Para ello, es necesario comprender la conceptualización de la marca empleador y de los servicios de operación logística, así como asociar las dimensiones y factores de la gestión del mismo con la edad, el género y el nivel jerárquico del talento humano de las organizaciones que comercializan servicios de operación logística en la ciudad de Bogotá, y finalmente identificar sus beneficios para la utilidad en sectores productivos que involucren a las organizaciones que internacionalizan esta actividad económica en la capital colombiana. 


\section{El employer branding en las organizaciones}

El branding es un concepto desde el cual las organizaciones gestionan su posicionamiento en la mente del cliente -ya sea interno o externo- de aquello que representa la marca y sus características diferenciadoras en comparación a otras existentes en el mercado (Jayasinghe, 2017); y desde este postulado las empresas deben generar valor a sus diferentes stakeholders, y uno de ellos son sus colaboradores. Esta necesidad de fidelizar a los empleados surge a partir del employer branding definido como "el proceso de construcción de la marca del empleador donde se aplican los principios de marketing a las actividades de recursos humanos dirigidas a los empleados actuales o potenciales de la empresa" (Dabirian, Paschen y Kietzmann, 2019, p. 85). Dicha construcción de marca como lo resaltan Rani y Kumar (2011) permitirá la obtención de reconocimientos públicos convirtiendo así a las organizaciones en entidades atractivas para trabajar, beneficiando la reputación general del empleador desde la satisfacción laboral del colaborador quienes fomentarán dicho conocimiento. Esta satisfacción es entendida como "la comparación entre el trabajo real y las expectativas que el trabajador se había generado en su cargo" (Sánchez-Sellero, M., Sánchez-Sellero, P., Cruz-González y Sánchez-Sellero, F. 2018, p. 5).

Ergun y Tatar (2018) consideran al employer branding como una decisión estratégica efectiva, la cual agrega valor a las organizaciones al permitir vincular el talento y el capital humano más valioso de éstas a su estructura organizacional, no sólo para el beneficio de la administración del personal sino de los resultados comerciales, financieros y operativos (Blasco, et al, 2014); por ello, a pesar que durante muchos años, la administración del talento humano se ha visualizado como una actividad operativa, económica y legal (García, Barros-Arrieta y Valle-Ospino, 2018), la perspectiva de relacionamiento con el consumidor ha visto la necesidad de establecer relaciones simbólicas y hasta emocionales entre la organización y su empleado (Ortegón-Cortázar y Royo-Vela, 2017).

Para que la naturaleza del employer branding tenga éxito, las organizaciones deben tener identificada y ejecutada la filosofía de orientación y conocimiento del cliente y del servicio (Sempértegui y Bravo, 2018; Vega y Serna, 2017), esto debido al hecho de que las organizaciones con problemas de motivación en beneficio de sus empleados, están impactando negativamente y de forma directa la atención a los clientes externos, y su propuesta de valor (Ergun y Tatar, 2018), efecto que se transforma en niveles de incertidumbre y deterioro relacional con proveedores, canales de distribución y otros grupos de interés (Robledo, Arán y Pérez-Aranda, 2015).

Todo proyecto de employer branding significativo lleva consigo un componente emocional, similar al consumer engagement generado desde la gestión de marca convencional (Bilińska-Reformat y Stańczyk, 2018) y con el fin de alcanzar los objetivos corporativos dicho 
vínculo emocional debe establecer un compromiso mutuo entre la organización y el empleado convirtiéndose así en un relacionamiento estratégico, génesis de ventajas competitivas únicas (Fernández-Lores, et al, 2014), la cuales repercuten en el desarrollo comercial, esto desde lo propuesto por Rani y Kumar (2011) cuando dicen que "el employer branding pone el foco en la venta desde dos perspectivas: la venta interna hacia el empleado de la propuesta de valor que ofrece la empresa y la venta externa hacia el mercado laboral de referencia" (p. 293).

Elementos del capital humano como lo son las habilidades, el conocimiento y la experiencia de las personas tienen un valor económico significativo para las organizaciones (Ergun y Tatar, 2018), los cuales proporcionan beneficios funcionales, económicos y psicológicos como propuesta de valor al empleado (Fernández-Lores, et al, 2014). Lo anterior sustenta la pertinencia de seis dimensiones relevantes, propuestas por Cardona-Arbeláez, et al, (2017) que permiten que el employer branding funcione asertivamente en una organización: contratación de personal, capacitación del personal, comunicaciones internas, motivación del personal, seguridad en el empleo y retención del personal.

Teniendo en cuenta que los empleados esperan que la empresa donde laboran les ofrezca oportunidades de crecimiento (Bednarska-Olejniczak, 2015) cada uno de dichos programas deben estar segmentados, porque los beneficios esperados por los diferentes grupos de empleados no son similares (Ergun y Tatar, 2018; Jayasinghe, 2017), por tanto, comprender las diferencias entre los segmentos del capital humano en las organizaciones permite optimizar las estrategias de contratación y retención para cada uno de ellos (Hernández, Espinoza y Aguilar, 2016).

Fuentes-Jiménez (2009) propone un modelo de gestión para el desarrollo del employer branding en las organizaciones, el cual se fundamenta en la estructuración de cinco dimensiones con sus respectivos factores. La dimensión de desarrollo está conformada por los factores de desarrollo de habilidades, orientación al cliente y nuevos conocimientos; la dimensión de contratación y retención la conforman factores como el reclutamiento, la selección y los reconocimientos, la dimensión de adecuación al trabajo está conformada por el ajuste de motivaciones, habilidades y el empowerment; la comunicación interna como dimensión contiene los objetivos y metas de la organización, los resultados alcanzados, la axiología corporativa y los cambios organizacionales; finalmente, la dimensión de comunicación externa la conforman factores como la organización en el macroambiente, la administración del portafolio de productos y el protagonismo en los medios.

\section{Los operadores logísticos}

Teniendo en cuenta que el crecimiento organizacional presenta una relación directa con el desarrollo de la cadena de suministro para la satisfacción de clientes, fabricantes, transportistas 
y todos los demás stakeholders (Govindan, Soleimani y Kannan, 2015) los operadores logísticos son un eslabón que potencializa actividades de gestión integral como lo son el manejo de los inventarios, al almacenamiento, la producción y la distribución (Olivos, Carrasco, Flores, Moreno y Nava, 2015). Este tipo de organizaciones se pueden visualizar como proveedores de servicios especializados en desarrollar el proceso administrativo de las actividades logísticas relacionadas con la cadena de suministro (Galo, Ribeiro, Mergulhão y Vidal, 2018).

Los procesos de globalización, de apertura económica y de dinamismo comercial a nivel internacional se convirtieron, entre otras, en las causas para que a finales del siglo XX los operadores logísticos empezaran a tener un rol altamente estratégico en el comercio exterior (Valencia y Bernal, 2017). Estas organizaciones poseen la capacidad de desarrollar diversas actividades en la cadena de suministro, como son el transporte, el almacenamiento, la distribución y la gestión de inventario, entre otras (López Rodríguez, Acosta Molina, Páez León, Sarmiento Páez y Tafur Serrano, 2018). Los operadores logísticos pueden desarrollar dos variables fundamentales desde las ópticas de gestión en la cadena logística, y de acuerdo a Orjuela, Ocampo y Bulla (2005) la primera es la gestión integral donde se llevan a cabo todas las funciones propias de la logística, desde el aprovisionamiento hasta la distribución física, y la segunda es la gestión parcial donde el operador se especializa en solo uno de los eslabones de la cadena de suministro.

El sector logístico ha resaltado diversas premisas a favor de su evolución durante los años venideros, dentro de las cuales Narváez-Pereda (2018) destacó el mercado de operadores logísticos, la distribución geográfica, la subcontratación y la importancia emergente de los sistemas de información empresarial. Las dinámicas tecnológicas que se incorporan a las diversas navieras y sistemas portuarios han permitido al sector logístico incorporar actividades de distribución y packing acordes al crecimiento del comercio internacional (Delfín-Ortega y Lenin-Navarro, 2015). Las organizaciones que se especializan en comercializar servicios de operación logística se enfocan en el manejo físico de mercancías, intermediación comercial, gestión y plataformas logísticas (Orjuela, et al, 2005), actividades que desde la óptica de Olivos, et al, (2015) dinamizan el desarrollo del sector privado y el crecimiento de diversos actores económicos de un estado o región.

Es relevante resaltar cómo, desde las actividades logísticas estos operadores pueden presentar diferentes tipos de modalidades de servicios; al respecto, dichas modalidades se presentan en la tabla 1. 
Tabla 1

Modalidades de servicio de los operadores logísticos

\begin{tabular}{|c|c|c|}
\hline Clasificación & Modalidad & Descripción \\
\hline \multirow{2}{*}{$\begin{array}{l}\text { De acuerdo con el tipo } \\
\text { de proceso }\end{array}$} & Logística dedicada & $\begin{array}{l}\text { Esta modalidad se presenta cuando el servicio logístico } \\
\text { se ofrece de manera exclusiva a un cliente, posterior a un } \\
\text { estudio de necesidades particulares. }\end{array}$ \\
\hline & Logística compartida & $\begin{array}{l}\text { Cuando se presenta esta modalidad los servicios ofrecidos } \\
\text { forman parte de un catálogo propio del operador logísti- } \\
\text { co, el cual puede ser demandado por múltiples empresas. }\end{array}$ \\
\hline \multirow{2}{*}{$\begin{array}{l}\text { De acuerdo con la locali- } \\
\text { zación o ubicación física } \\
\text { de la operación }\end{array}$} & Logística en la empresa & $\begin{array}{l}\text { Esta modalidad se desarrolla directamente en las áreas de } \\
\text { gestión de la empresa que contrata el servicio, otorgando } \\
\text { infraestructura propia al operador logístico para la ejecu- } \\
\text { ción del contrato. }\end{array}$ \\
\hline & $\begin{array}{l}\text { Logística fuera de la em- } \\
\text { presa }\end{array}$ & $\begin{array}{l}\text { Desde esta modalidad el servicio se desarrolla en las ins- } \\
\text { talaciones del operador logístico, el cual debe tener un } \\
\text { domicilio diferente al del cliente. }\end{array}$ \\
\hline \multirow{2}{*}{$\begin{array}{l}\text { De acuerdo con los re- } \\
\text { cursos humanos y téc- } \\
\text { nicos }\end{array}$} & Recursos propios & $\begin{array}{l}\text { En esta modalidad se emplean los recursos del cliente de } \\
\text { manera total o temporal, mientras que la gestión del ser- } \\
\text { vicio es utilizado por el operador logístico. }\end{array}$ \\
\hline & Recursos externos & $\begin{array}{l}\text { Cuando se presenta esta modalidad los recursos para el } \\
\text { desarrollo de los servicios logísticos pertenecen a la com- } \\
\text { pañía al operador y no a los clientes. }\end{array}$ \\
\hline
\end{tabular}

Fuente: Elaboración propia adaptado de Orjuela, et al, (2005)

\section{Metodología}

Se utilizó la prueba estadística Chi Cuadrado de Pearson, que es un sistema estadístico no paramétrico utilizado para pruebas de significación de la hipótesis nula de independencia estadística entre variables de categorías en tablas de contingencia (Gómez, 2008). La muestra estuvo conformada por 384 empleados de organizaciones que internacionalizan servicios de operación logística en la ciudad de Bogotá, Colombia. Los participantes fueron seleccionados desde un muestreo no probabilístico por conveniencia.

El instrumento para el levantamiento de la información fue una encuesta, construida a partir del modelo de gestión del employer branding propuesto por Fuentes-Jiménez (2009) la cual está dividida en dos partes: la primera presenta siete ítems que tienen como finalidad caracterizar sociodemográficamente a los participantes por medio de preguntas de tipo nominal; la segunda parte presenta veinticuatro ítems que caracterizan el modelo de gestión del employer branding estudiado por medio de preguntas tipo Likert organizadas en cinco dimensiones con sus respectivos factores: desarrollo (capacitación, información del cliente, 
cliente externo y conocimiento), contratación y retención (reclutamiento, selección, nuevos empleados, pagos extras y reconocimientos), adecuación al trabajo (funciones, habilidades, desarrollo de actividades, necesidades del cliente interno), comunicación interna (metas y objetivos, alcance de resultados, oportunidad de expresión y axiología corporativa) y comunicación externa (la organización en el macroambiente, administración del portafolio de productos y protagonismo en los medios).

El levantamiento de la información se llevó a cabo en el primer trimestre del año 2019 y la validación del instrumento se desarrolló con una prueba piloto del $10 \%$ de la muestra a través de la prueba Alfa de Cronbach obteniendo resultados satisfactorios para cada una de sus dimensiones tal como lo plasma la tabla 2. Este análisis estadístico se desarrolló utilizando el paquete estadístico SPSS versión 25.0.

Tabla 2

Estadísticas de fiabilidad para cada dimensión del instrumento

\begin{tabular}{lccc}
\hline Dimensiones del instrumento & Alfa de Cronbach & $\begin{array}{c}\text { Alfa de Cronbach basada en } \\
\text { elementos estandarizados }\end{array}$ & $\begin{array}{c}\text { Número de } \\
\text { elementos }\end{array}$ \\
\hline Desarrollo & 0.791 & 0.792 & 4 \\
Contratación y retención & 0.802 & 0.801 & 5 \\
Adecuación al trabajo & 0.875 & 0.882 & 4 \\
Comunicación interna & 0.823 & 0.824 & 7 \\
Comunicación externa & 0.807 & 0.808 & 4 \\
\hline
\end{tabular}

Fuente: Elaboración propia

\section{Resultados}

Las edades de los participantes estuvieron entre los 18 a los 52 años. La distribución del género fue de $51.2 \%$ mujeres y de un $46.5 \%$ hombres. El 5.5\% posee una formación de bachillerato básico, el $70.7 \%$ técnica y tecnológica y el $23.4 \%$ formación profesional y a nivel de especialización un $0.4 \%$. Frente a su condición laboral en la empresa a la que pertenecen el $56.9 \%$ posee un contrato laboral a término indefinido, mientras que el $33.5 \%$ posee un contrato a término fijo. Finalmente, el $59.7 \%$ de la población hace parte del nivel operativo de la organización, el $29.9 \%$ a la media gerencia y el $10.4 \%$ a la alta gerencia.

Se identificaron los factores de las dimensiones del modelo de gestión del employer branding (Fuentes-Jiménez, 2009) con los cuales existe un significativo nivel de asociación con la edad, el género y el nivel jerárquico al interior de las organizaciones de la población estudiada, 
esto sustentado por Horstmanny y Martín (2017) cuando afirman que el nuevo milenio se caracteriza por la escasez del talento, el cual implica desafíos organizacionales en los procesos de reclutamiento y selección debido a los factores sociodemográficos del mercado laboral. Teniendo en cuenta que la significación asintótica (bilateral) desde Chi-cuadrado de Pearson en los factores de las dimensiones encontradas son inferiores a 0.05 se rechaza la hipótesis nula y a su vez se acepta la hipótesis alternativa, encontrando relaciones significativas a un nivel del $95 \%$ de confiabilidad y un 5\% de error (Páramo-Morales, 2015). Dichas asociaciones se observan en la tabla 3.

A continuación, se presenta un análisis de correspondencias simples y la normalización simétrica para los diferentes resultados obtenidos en la tabla 3. El análisis de correspondencias simples resume cantidades importantes de información en un número reducido de dimensiones (Fontalvo-Cerpa, 2015); y para este caso, entre las dos primeras dimensiones existe una contribución a la inercia total superior al $80.0 \%$ sustentando que las dependencias observadas están adecuadamente recogidas, comprobando así que las variables no son independientes (Sánchez-Sellero, et al, 2018). Con el desarrollo del método de normalización simétrica se identificaron y analizaron las semejanzas y diferencias entre la edad de los participantes, su género y su nivel jerárquico en la organización frente a los factores de las dimensiones del modelo de gestión del employer branding (Fuentes-Jiménez, 2009). En el desarrollo del método de normalización simétrica "para cada dimensión, las puntuaciones de fila son la media ponderada de las puntuaciones de columna divididas por el valor propio coincidente, y al revés para las puntuaciones de columna" (Sánchez-Sellero, et al, 2018, p. 12). 
Tabla 3

Resultados estadísticos de asociación en los factores de las dimensiones del modelo de gestión del employer branding

\begin{tabular}{|c|c|c|}
\hline Variables & $\begin{array}{l}\text { Factores de las dimensiones del modelo de ges- } \\
\text { tión del employer branding }\end{array}$ & $\begin{array}{l}\text { Significación asintótica (bilateral) } \\
\text { desde Chi-cuadrado de Pearson }\end{array}$ \\
\hline \multirow{5}{*}{ Edad } & $\begin{array}{l}\text { Recibo información respecto a la exigencia de los } \\
\text { clientes de la empresa }\end{array}$ & 0.000 \\
\hline & $\begin{array}{l}\text { Las actividades y responsabilidades de los nuevos } \\
\text { colaboradores son claramente definidas. }\end{array}$ & 0.001 \\
\hline & $\begin{array}{l}\text { Soy remunerado de forma justa de acuerdo con } \\
\text { mis competencias y formación. }\end{array}$ & 0.048 \\
\hline & $\begin{array}{l}\text { La empresa ofrece la oportunidad de recibir ingre- } \\
\text { sos extras. }\end{array}$ & 0.009 \\
\hline & $\begin{array}{l}\text { Existen en la empresa reconocimientos a los cola- } \\
\text { boradores por sus logros. }\end{array}$ & 0.024 \\
\hline \multirow{8}{*}{ Género } & $\begin{array}{l}\text { La empresa me capacita para mejorar los procesos } \\
\text { que desarrollo dentro de ella. }\end{array}$ & 0.031 \\
\hline & $\begin{array}{l}\text { Las actividades y responsabilidades de los nuevos } \\
\text { colaboradores son claramente definidas. }\end{array}$ & 0.020 \\
\hline & $\begin{array}{l}\text { La empresa ofrece la oportunidad de recibir ingre- } \\
\text { sos extras. }\end{array}$ & 0.021 \\
\hline & $\begin{array}{l}\text { Existen en la empresa reconocimientos a los cola- } \\
\text { boradores por sus logros. }\end{array}$ & 0.018 \\
\hline & $\begin{array}{l}\text { Si el colaborador desea, él puede solicitar cambio } \\
\text { de función y de área de gestión. }\end{array}$ & 0.014 \\
\hline & $\begin{array}{l}\text { Las metas y los objetivos de la empresa son divul- } \\
\text { gados a todos los colaboradores. }\end{array}$ & 0.001 \\
\hline & Conozco los resultados generales de la empresa. & 0.013 \\
\hline & $\begin{array}{l}\text { Las amenazas que llegan a la empresa son comu- } \\
\text { nicadas a los colaboradores. }\end{array}$ & 0.021 \\
\hline \multirow{4}{*}{$\begin{array}{c}\text { Nivel jerárquico } \\
\text { en la } \\
\text { organización }\end{array}$} & $\begin{array}{l}\text { Recibo información respecto a la exigencia de los } \\
\text { clientes de la empresa }\end{array}$ & 0.012 \\
\hline & $\begin{array}{l}\text { La empresa me ofrece oportunidades para aumen- } \\
\text { tar mi conocimiento a su favor. }\end{array}$ & 0.049 \\
\hline & $\begin{array}{l}\text { Existen en la empresa reconocimientos a los cola- } \\
\text { boradores por sus logros. }\end{array}$ & 0.039 \\
\hline & $\begin{array}{l}\text { Los cambios que van a ocurrir a nivel general en } \\
\text { la empresa son comunicados con anticipación a } \\
\text { todos los colaboradores. }\end{array}$ & 0.013 \\
\hline
\end{tabular}

Fuente: Elaboración propia. 
Tabla 4

Análisis de correspondencias simples entre la edad del cliente interno y su perspectiva frente a la justa remuneración en la empresa

\begin{tabular}{|c|c|c|c|c|c|c|c|c|}
\hline Dimensión & $\begin{array}{l}\text { Valor } \\
\text { singular }\end{array}$ & Inercia & $\begin{array}{c}\text { Chi } \\
\text { cuadrado }\end{array}$ & Sig. & cuntavinzauv & Acunnulaue & \multicolumn{2}{|c|}{$\begin{array}{c}\text { Valor singular de } \\
\text { confianza } \\
\text { Desviación Correlación }\end{array}$} \\
\hline 1 & .210 & .044 & & & .539 & .539 & .048 & .084 \\
\hline 2 & .167 & .028 & & & .341 & .880 & .048 & \\
\hline 3 & .096 & .009 & & & .111 & .992 & & \\
\hline 4 & .026 & .001 & & & .008 & 1.000 & & \\
\hline Total & & .082 & 31.604 & $.137^{\mathrm{a}}$ & 1.000 & 1.000 & & \\
\hline a. 24 grados & de libertad & & & & & & & \\
\hline
\end{tabular}

Fuente: Elaboración propia

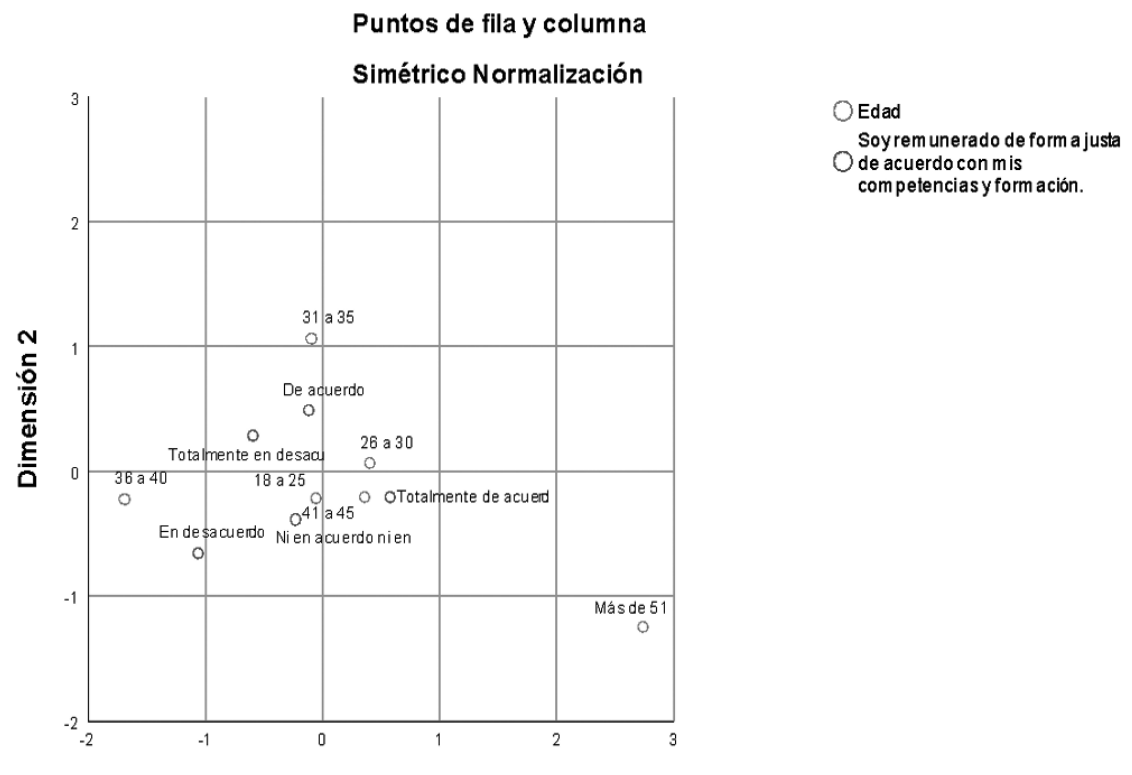

Figura 1. Normalización simétrica entre la edad del cliente interno y su perspectiva frente a la justa remuneración en la empresa.

Fuente: Elaboración propia. 
El resultado obtenido y evidenciado en la tabla 4 demuestra que entre la edad del cliente interno de las organizaciones estudiadas, y su perspectiva frente a la justa remuneración en la empresa existe un nivel de explicación de los datos sobre dos dimensiones con una inercia del $88.0 \%$ en términos de análisis de correspondencias simples en reducción de dimensiones. Así mismo, desde lo visualizado en la figura 1 los empleados con edades entre los 18 a 30 años están totalmente de acuerdo con afirmar que son remunerados de forma justa de acuerdo con sus competencias y formación. Los de 31 a 35 años están de acuerdo con la misma afirmación, mientras que las personas con edades entre los 36 a 40 años manifiestan su desacuerdo con esta afirmación. Los colaboradores con edades entre los 41 a los 45 años aseveran respecto a dicha afirmación no estar ni de acuerdo ni en desacuerdo.

Tabla 5

Análisis de correspondencias simples entre la edad del cliente interno y su perspectiva frente a la existencia de reconocimientos a colaboradores en la empresa

\begin{tabular}{|c|c|c|c|c|c|c|c|c|}
\hline \multirow[b]{2}{*}{ Dimensión } & \multirow[b]{2}{*}{$\begin{array}{l}\text { Valor } \\
\text { singular }\end{array}$} & \multirow[b]{2}{*}{ Inercia } & \multirow[b]{2}{*}{ Chi cuadrado } & \multirow[b]{2}{*}{ Sig. } & \multicolumn{2}{|c|}{ Proporción de inercia } & \multicolumn{2}{|c|}{ Valor singular de confianza } \\
\hline & & & & & Contabilizado & Acumulado & $\begin{array}{l}\text { Desviación } \\
\text { estándar }\end{array}$ & $\begin{array}{c}\text { Correlación } \\
2\end{array}$ \\
\hline 1 & .231 & .054 & & & .601 & .601 & .045 & -.100 \\
\hline 2 & .163 & .027 & & & .298 & .900 & .046 & \\
\hline 3 & .093 & .009 & & & .097 & .997 & & \\
\hline 4 & .017 & .000 & & & .003 & 1.000 & & \\
\hline Total & & .089 & 34.296 & $.080^{\mathrm{a}}$ & 1.000 & 1.000 & & \\
\hline \multicolumn{9}{|c|}{ a. 24 grados de libertad } \\
\hline
\end{tabular}

Fuente: Elaboración propia

La tabla 5 muestra que entre la edad de los empleados, y su perspectiva frente a la existencia de reconocimientos a colaboradores en la empresa existe un nivel de explicación de los datos sobre dos dimensiones con una inercia del $90.0 \%$ en términos de análisis de correspondencias simples en reducción de dimensiones. Los colaboradores con edades entre los 26 a 30 años están totalmente de acuerdo con afirmar que existen en la empresa reconocimientos por sus logros, a diferencia del cliente interno con edades entre los 36 a 40 quienes están totalmente en desacuerdo. Los empleados con edades entre los 31 a 35 años están de acuerdo con esta afirmación; lo anterior reflejado en la figura 2. 


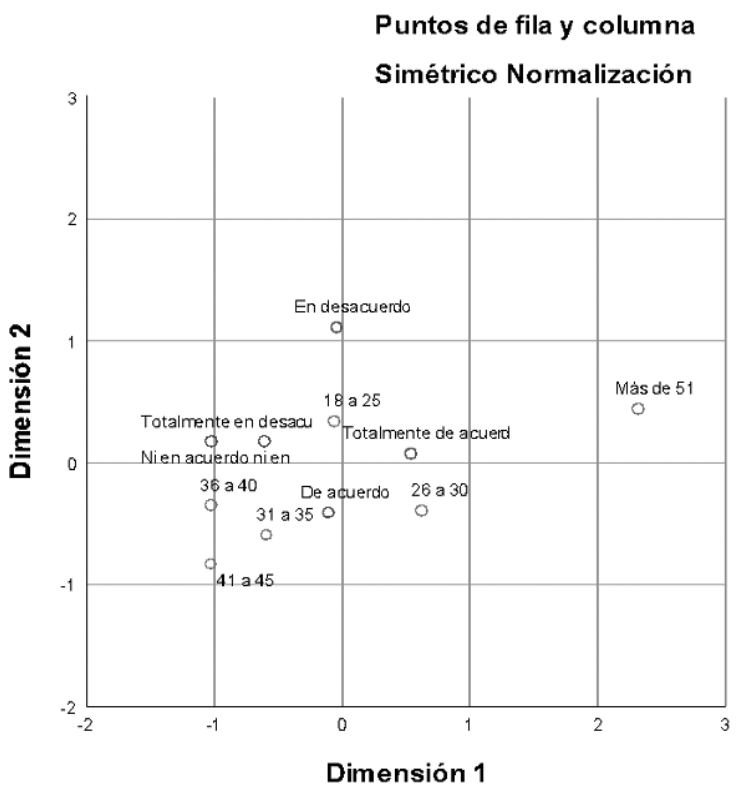

Edad

Existen en la empresa Oreconocimientos alos colaboradorespor suslogros.

Figura 2. Normalización simétrica entre la edad del cliente interno y su perspectiva frente a la existencia de reconocimientos a colaboradores en la empresa.

Fuente: Elaboración propia

Tabla 6

Análisis de correspondencias simples entre el género de los nuevos colaboradores y la identificación de sus responsabilidades al vincularse laboralmente

\begin{tabular}{|c|c|c|c|c|c|c|c|c|}
\hline \multirow[b]{2}{*}{ Dimensión } & \multirow[b]{2}{*}{$\begin{array}{c}\text { Valor } \\
\text { singular }\end{array}$} & \multirow[b]{2}{*}{ Inercia } & \multirow[b]{2}{*}{$\begin{array}{c}\text { Chi } \\
\text { cuadrado }\end{array}$} & \multirow[b]{2}{*}{ Sig. } & \multicolumn{2}{|c|}{ Proporción de inercia } & \multicolumn{2}{|c|}{ Valor singular de confianza } \\
\hline & & & & & Contabilizado & Acumulado & $\begin{array}{c}\text { Desviación } \\
\text { estándar }\end{array}$ & $\begin{array}{c}\text { Correlación } \\
2\end{array}$ \\
\hline 1 & .207 & .043 & & & .914 & .914 & .044 & -.043 \\
\hline 2 & .064 & .004 & & & .086 & 1.000 & .048 & \\
\hline Total & & .047 & 18.112 & $.020^{\mathrm{a}}$ & 1.000 & 1.000 & & \\
\hline & a. $8 \mathrm{grad}$ & de libert & & & & & & \\
\hline
\end{tabular}

Fuente: Elaboración propia

Se observa que entre el género de los nuevos colaboradores y la identificación de sus responsabilidades al vincularse laboralmente existe un nivel de explicación de los datos sobre dos dimensiones con una inercia del $100.0 \%$ en términos de análisis de correspondencias simples 
en reducción de dimensiones, esto de acuerdo a la tabla 6. Así mismo, la figura 3 describe que los colaboradores de género masculino están totalmente de acuerdo con afirmar que las actividades y responsabilidades de los nuevos colaboradores son claramente definidas; mientras que el género femenino no está ni de acuerdo ni en desacuerdo con esta afirmación.

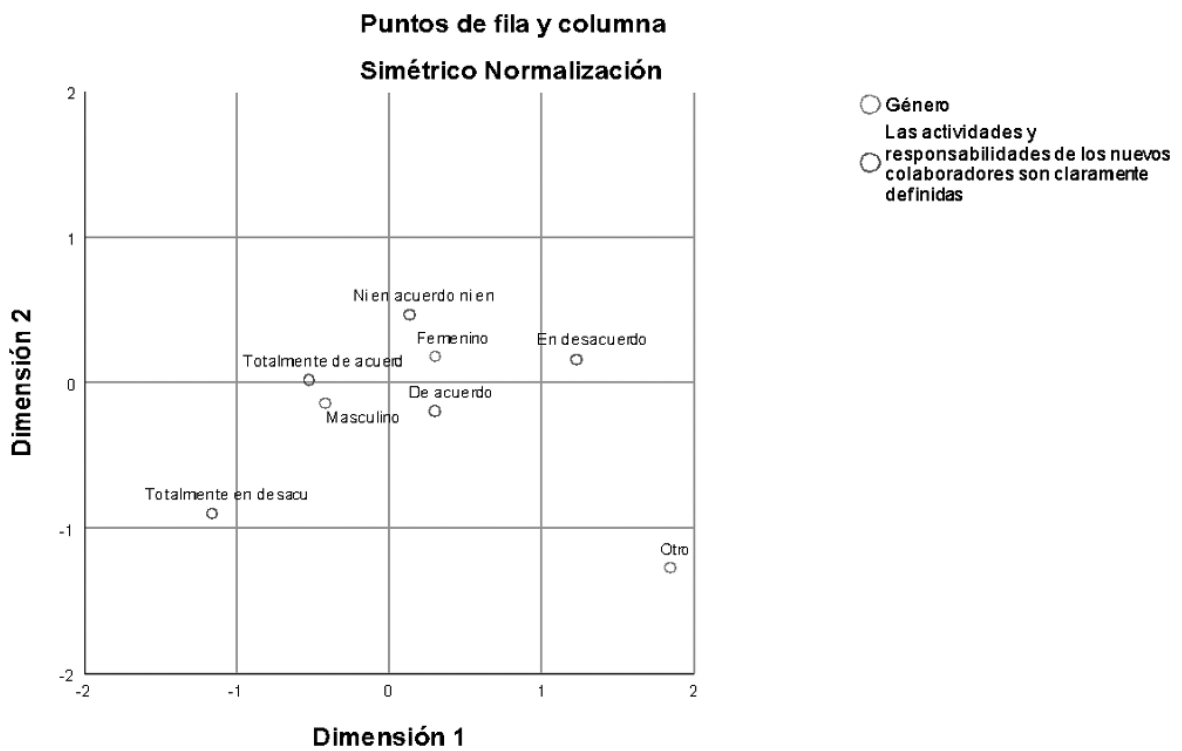

Figura 3. Normalización simétrica entre el género de los nuevos colaboradores y la identificación de sus responsabilidades al vincularse laboralmente.

Fuente: Elaboración propia.

Tabla 7

Análisis de correspondencias simples entre el género del cliente interno y su perspectiva frente a la oportunidad de recibir ingresos extras en las empresas

\begin{tabular}{|c|c|c|c|c|c|c|c|c|}
\hline \multirow[b]{2}{*}{ Dimensión } & \multirow[b]{2}{*}{ Valor singular } & \multirow[b]{2}{*}{ Inercia } & \multirow[b]{2}{*}{ Chi cuadrado } & \multirow[b]{2}{*}{ Sig. } & \multicolumn{2}{|c|}{ Proporeión de inereia } & \multicolumn{2}{|c|}{ Valor singular de eonfianza } \\
\hline & & & & & Contabilizado & Acumulado & $\begin{array}{c}\text { Desviación } \\
\text { estándar }\end{array}$ & $\begin{array}{c}\text { Correlación } \\
2\end{array}$ \\
\hline 1 & .178 & .032 & & & 678 & 678 & .048 & .095 \\
\hline 2 & .123 & .015 & & & .322 & 1.000 & .058 & \\
\hline Total & & .047 & 18.036 & $.021^{\mathrm{a}}$ & 1.000 & 1.000 & & \\
\hline a. 8 grados & libertad & & & & & & & \\
\hline
\end{tabular}

Fuente: Elaboración propia 

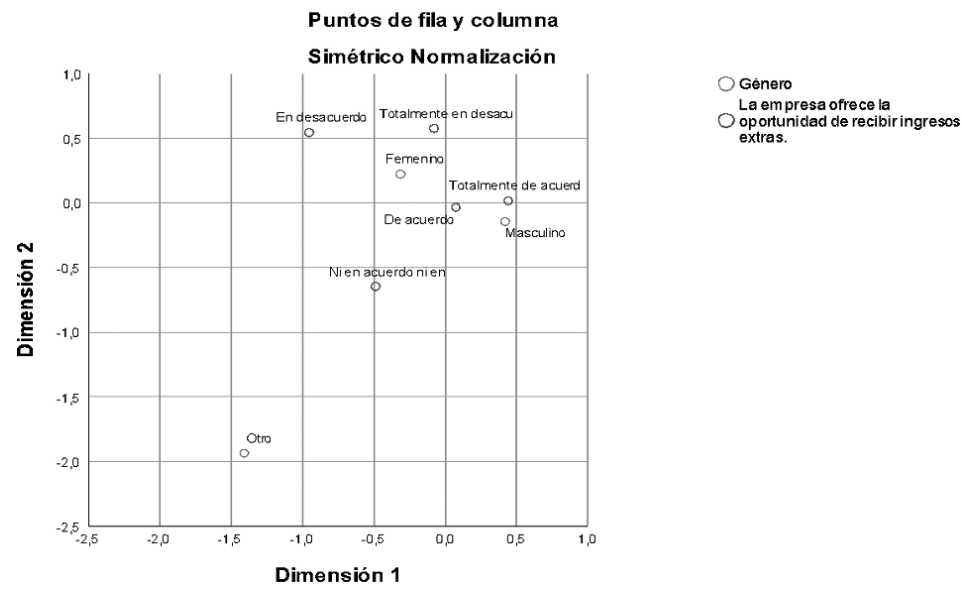

Figura 4. Normalización simétrica entre el género del cliente interno y su perspectiva frente a la oportunidad de recibir ingresos extras en las empresas.

Fuente: Elaboración propia.

La tabla 7 determina que entre el género del cliente interno y su perspectiva frente a la oportunidad de recibir ingresos extras en las empresas existe un nivel de explicación de los datos sobre dos dimensiones con una inercia del $100.0 \%$ en términos de análisis de correspondencias simples en reducción de dimensiones. En la figura 4 se evidencia que los colaboradores de género masculino están totalmente de acuerdo con afirmar que la empresa ofrece la oportunidad de recibir ingresos extras, contrario a lo manifestado por el género femenino que revela que ante dicha afirmación está totalmente en desacuerdo.

Se observa que entre el género de los colaboradores y la posibilidad de solicitar cambio de labores en la empresa existe un nivel de explicación de los datos sobre dos dimensiones con una inercia del $100.0 \%$ en términos de análisis de correspondencias simples en reducción de dimensiones, esto de acuerdo a la tabla 8. Así mismo, la figura 5 puntualiza que los colaboradores de género masculino están de acuerdo con afirmar que, si el colaborador desea, él puede solicitar cambio de funciones, así como de área de gestión; por el contrario, el género femenino está en desacuerdo con esto. 
Tabla 8

Análisis de correspondencias simples entre el género del colaborador y su posibilidad de solicitar cambio de labores en la empresa

\begin{tabular}{|c|c|c|c|c|c|c|c|c|}
\hline \multirow[b]{2}{*}{ Dimensión } & \multirow[b]{2}{*}{$\begin{array}{l}\text { Valor } \\
\text { singular }\end{array}$} & \multirow[b]{2}{*}{ Inercia } & \multirow[b]{2}{*}{ Chi cuadrado } & \multirow[b]{2}{*}{ Sig. } & \multicolumn{2}{|c|}{ Proporción de inercia } & \multicolumn{2}{|c|}{ Valor singular de confianza } \\
\hline & & & & & \multicolumn{2}{|c|}{ Contabilizado Acumulado } & $\begin{array}{l}\text { Desviación } \\
\text { estándar }\end{array}$ & $\begin{array}{c}\text { Correlación } \\
2\end{array}$ \\
\hline 1 & .197 & .039 & & & .779 & .779 & .048 & .068 \\
\hline 2 & .105 & .011 & & & .221 & 1.000 & .065 & \\
\hline \multicolumn{2}{|l|}{ Total } & .050 & 19.200 & $.014^{\mathrm{a}}$ & 1.000 & 1.000 & & \\
\hline \multicolumn{9}{|c|}{ a. 8 grados de libertad } \\
\hline
\end{tabular}

Fuente: Elaboración propia

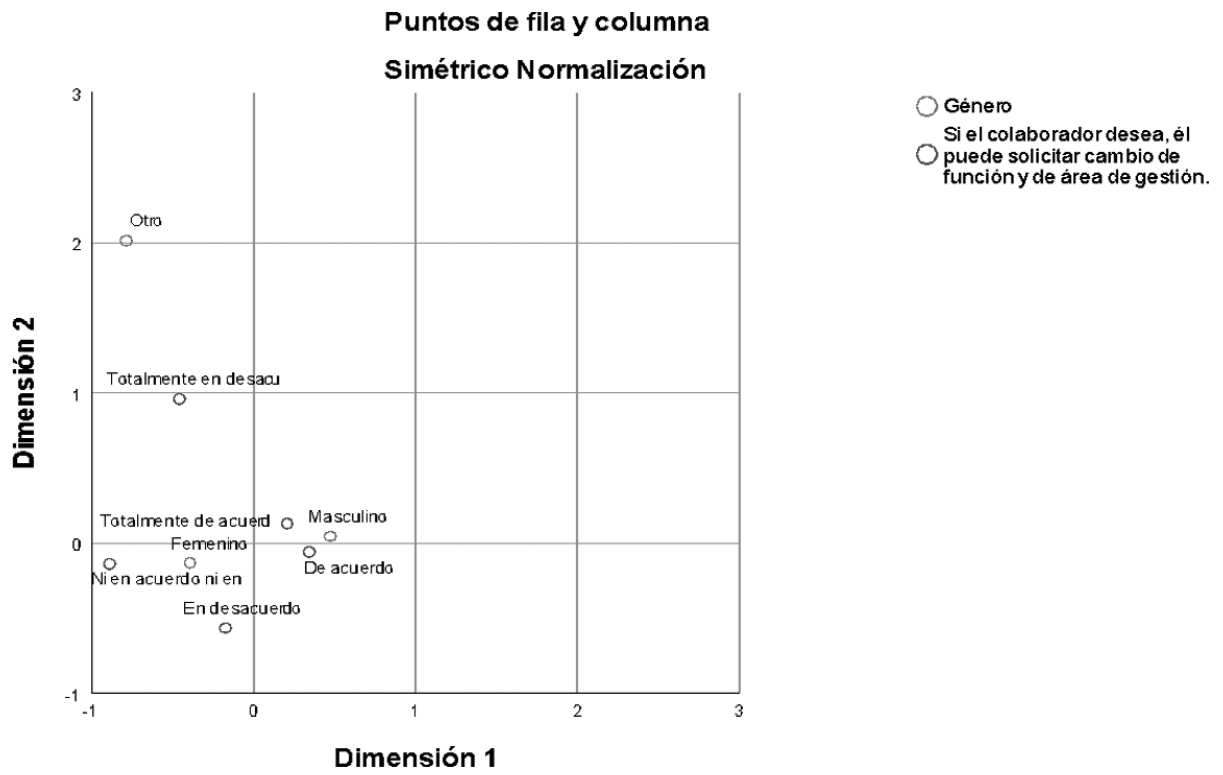

Figura 5. Normalización simétrica entre el género del colaborador y su posibilidad de solicitar cambio de labores en la empresa.

Fuente: Elaboración propia. 
Tabla 9

Análisis de correspondencias simples entre el nivel jerárquico del cliente interno en la organización y su perspectiva frente a la capacitación recibida en la empresa.

\begin{tabular}{ccccccccc}
\hline & & & & \multicolumn{2}{c}{ Proporción de inercia } & \multicolumn{2}{c}{ Valor singular de confianza } \\
Dimensión & $\begin{array}{c}\text { Valor } \\
\text { singular }\end{array}$ & Inercia & $\begin{array}{c}\text { Chi } \\
\text { cuadrado }\end{array}$ & Sig. & Contabilizado & Acumulado & $\begin{array}{c}\text { Desviación } \\
\text { estándar }\end{array}$ & Correlación \\
1 & .141 & .020 & & & .776 & .776 & .052 & .071 \\
\hline 2 & .076 & .006 & & & .224 & 1.000 & .037 \\
\multicolumn{2}{c}{ Total } & .025 & 9.585 & $.295^{\mathrm{a}}$ & 1.000 & 1.000 & & \\
a. 8 grados de libertad & & & & & & & \\
\hline
\end{tabular}

Fuente: Elaboración propia

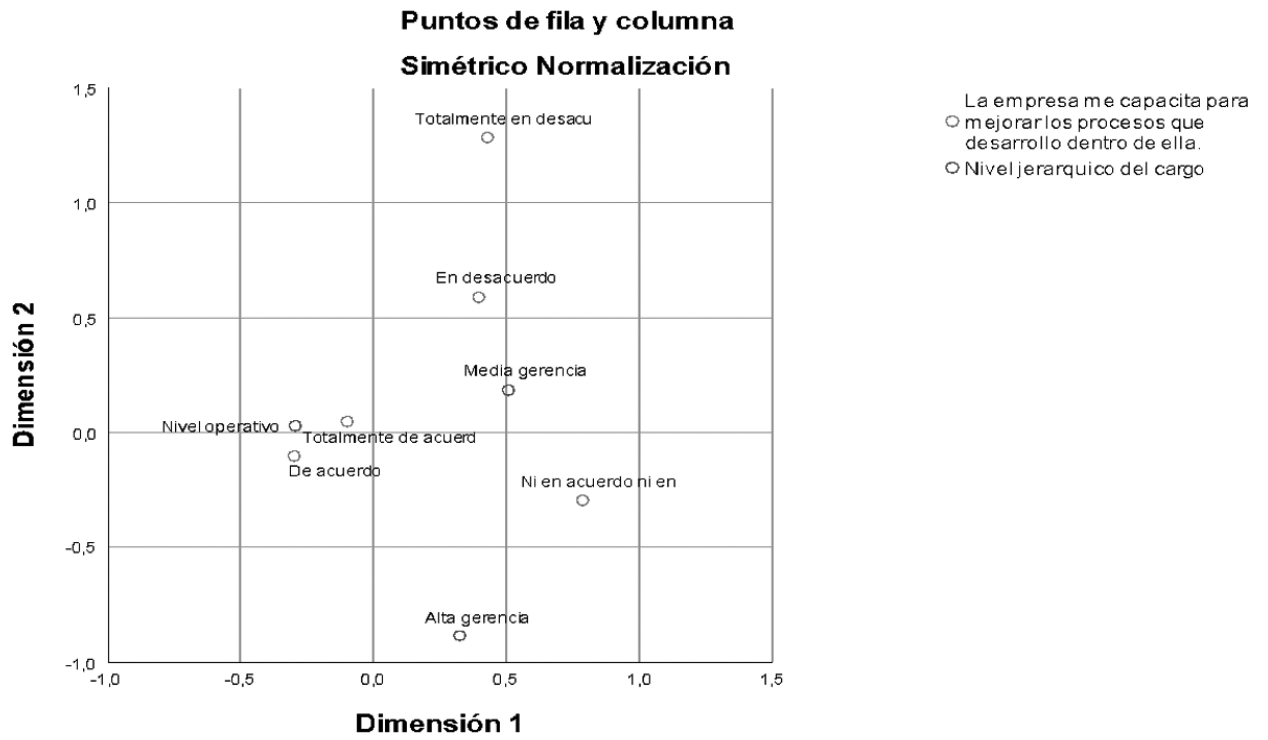

Figura 6. Normalización simétrica entre el nivel jerárquico del cliente interno en la organización y su perspectiva frente a la capacitación recibida en la empresa.

Fuente: Elaboración propia.

La tabla 9 determina que entre el nivel jerárquico del cliente interno en la organización y su perspectiva frente a la capacitación recibida en la empresa existe un nivel de explicación de los datos sobre dos dimensiones con una inercia del $100.0 \%$ en términos de análisis de correspondencias simples en reducción de dimensiones. En la figura 6 se observa que los 
colaboradores que pertenecen al nivel operativo están de acuerdo con afirmar que la empresa los capacita para mejorar los procesos que desarrollan dentro de ella, los que hacen parte de la media gerencia están en desacuerdo con esta afirmación, y los colaboradores que conforman la alta gerencia manifiestan no estar ni de acuerdo ni en desacuerdo con este hecho.

\section{Conclusiones}

El desarrollo y conocimiento de los empleados son algunas de las formas más eficientes para obtener una ventaja competitiva (Hwang, 2012); sin embargo las empresas participantes en este proceso investigativo se han centrado en capacitar a los empleados de nivel operativo, pero la percepción de los colaboradores de alta y media gerencia es la ausencia de esquemas de capacitación, siendo esto una oportunidad de mejoramiento para las organizaciones que internacionalizan servicios de operación logística de la capital colombiana, teniendo en cuenta que los empleados, sin importar su nivel de formación o grado de responsabilidad en la organización, deben tener la oportunidad y la percepción de que están desarrollando sus competencias, favoreciendo la posibilidad de mantener a los mejores talentos en las empresas (Theurer, Tumasjan, Welpe y Lievens, 2018).

El factor relacionado con la remuneración es uno de los principales intereses de los empleados desde la búsqueda de mejores salarios (Fuentes-Jiménez, 2009), aunque los colaboradores entre 18 y 35 años expusieron que en las empresas sí existen reconocimientos a su labor y la remuneración es justa, aunque las personas más adultas consideran no sentirse satisfechas con estos elementos. La posibilidad de generar ingresos extras puede mejorar la percepción de marca del empleador llevando a mayores niveles de retención del cliente interno (Bussin y Mouton, 2019); sin embargo, dicha realidad puede estar dada por la experiencia laboral, pues son los empleados más adultos los que consideran que no existe una remuneración adecuada porque deberían estar mejor remunerados, situación que sustenta la concepción de que la justa remuneración está dada no sólo por el cargo y la responsabilidad, sino también la percepción desde la experiencia del trabajador (Bussin y Mouton, 2019; Moroko y Uncles, 2009).

En lo referente a la segmentación del cliente interno, se encontró que empleados de género femenino se sentían más limitados a poder cambiar de labor según sus gustos e intereses, siendo esto completamente opuesto a lo manifestado por el género masculino. Frente a lo anterior, es importante resaltar la pertinencia de la segmentación por género en los procesos de employer branding, pues cada segmento posee expectativas diversas las cuales deben ser tenidas en cuenta por los empleadores en relación con la propuesta de valor ofrecida previamente en los procesos de reclutamiento, selección y contratación (Moroko y Uncles, 2009). 
Teniendo en cuenta los resultados obtenidos se resalta la necesidad de identificar y segmentar al cliente interno a partir de variables como la edad, el género y su nivel jerárquico en la organización, teniendo en cuenta los factores relacionados con las dimensiones del modelo de gestión del employer branding, en especial a elementos como el desarrollo, la contratación y retención, la adecuación al trabajo, la comunicación interna y externa, que potencializan la imagen de la marca empleador en el mercado laboral, no sólo en el sector de la internacionalización de servicios de operación logística, sino en otras actividades económicas.

Desde el reconocimiento de las anteriores perspectivas de los clientes internos en las organizaciones que internacionalizan servicios de operación logística en la ciudad de Bogotá Colombia, surge la inquietud de desarrollar procesos investigativos relacionados con la gestión del capital humano y el employer branding en otros sectores productivos, involucrando no sólo la percepción de los colaboradores sino de otros stakeholders, como los accionistas, los proveedores y los consumidores; de esta manera, se obtendrá un acercamiento sobre la orientación al mercado de las empresas, elemento que les permite estar preparadas para las dinámicas propias del actual escenario comercial, el cual está influenciado por la globalización, el desarrollo tecnológico, la responsabilidad social y la humanización (Neirotti y Pesce, 2019), elementos que deben direccionar la propuesta de valor de las organizaciones que desean incursionar en mercados internacionales, alcanzando desde la gestión desde la marca empleador un óptimo y privilegiado posicionamiento en el mercado laboral.

\section{Referencias}

Bednarska-Olejniczak, D. (2015). Employer Branding a CSR-bank jako pracodawca społecznie odpowiedzialny. Marketing i Zarządzanie, 41 (1), 237-250. https://doi.org/10.18276/pzfm.2015.41/1-19

Bilińska-Reformat, K., \& Stańczyk, I. (2018). Employer Branding as a Source of Competitive Advantage of Retail Chains. Journal of Management and Business Administration. Central Europe, 26(1), 2-12. https://doi. org/10.7206/jmba.ce.2450-7814.216

Bussin, M., \& Mouton, H. (2019). Effectiveness of employer branding on staff retention and compensation expectations. South African Journal of Economic and Management Sciences, 22(1), 1-8. http://dx.doi.org/10.4102/ sajems.v22i1.2412

Cardona-Arbelaez, D, Henriquez-Fuentes, G., Rada-Llanos, J., Martínez, J, y Bonnet, M. (2017). Endomarketing: Estrategia de innovación en las organizaciones el siglo XXI. Revista ESPACIOS, 38(57). https://doi. org/10.1108/09564239510078849

Dabirian, A., Paschen, J., \& Kietzmann, J. (2019). Employer Branding: Understanding Employer Attractiveness of IT Companies. IT Professional, 21(1), 82-89. https://doi.org/10.1109/mitp.2018.2876980

Delfín-Ortega, O. y Lenin-Navarro, J. (2015). Productividad total de los factores en las terminales de contenedores en los puertos de México: una medición a través del índice Malmquist. Contaduría y administración, 60(3), 663-685. http://dx.doi.org/10.1016/j.cya.2015.05.011

Departamento Nacional de Planeación - DPN (2016). Misión logística para Colombia. Recuperado el 24 de abril de 2019 de https://www.infraestructura.org.co/13congreso/memorias/24/SIMONGAVIRIA.pdf 
Ergun, H. \& Tatar, B. (2018). Employer branding and employee attitudes: mediating role of person-organization fit. Research Journal of Business and Management, 5(2), 110-120. https://doi.org/10.17261/pressacademia.2018.830

Fernández-Lores, S., Avelló, M., Gavilán, D., y Blasco, F. (2014). 18 años de employer branding: hacia una definición más precisa. International Journal of Communication Research, 10(10). https://doi.org/10.7263/adresic-010-02

Fontalvo-Cerpa, W. (2015). Análisis multivariado para determinar los factores más relevantes de deserción estudiantil presentes en el programa de Ingeniería Industrial de una Universidad del Caribe colombiano. Revista Prospectiva, 13(1), 86-98. https://doi.org/10.15665/rp.v13i1.363

Fuentes-Jiménez, P. (2009). Operativa del marketing interno: Propuesta de modelo de endomarketing. Perspectivas, (23), 189-231.

Galo, N., Ribeiro, P., Mergulhão, R. y Vidal, J. (2018). Selección de proveedor de servicios logísticos: alineación entre criterios e indicadores. Innovar: Revista de ciencias administrativas y sociales, 28(69), 55-70. https://doi. org/10.15446/innovar.v28n69.71696

García, E, Barros-Arrieta, D. y Valle-Ospino, A. (2018). Endomarketing desde el paradigma de la sostenibilidad organizacional. Desarrollo Gerencial, 10(2), 65-82. https://doi.org/10.17081/dege.10.2.3219

Gómez, O. (2008). Una aplicación de la prueba chi cuadrado con SPSS. Industrial data, 11(1), 73-77. https://doi. org/10.15381/idata.v11i1.6040

González Correa, J. (2015). Contratación logística en Colombia: implementación de un operador logístico integral. Semestre Económico, 18(38), 215-237. https://doi.org/10.22395/seec.v18n38a8

Govindan, K., Soleimani, H., y Kannan, D. (2015). Reverse logistics and closed-loop supply chain: A comprehensive review to explore the future. European Journal of Operational Research, 240(3), 603-626. https://doi. org/10.22395/seec.v18n38a8

Hadi, N., \& Ahmed, S. (2018). Role of Employer Branding Dimensions on Employee Retention: Evidence from Educational Sector. Administrative Sciences, 8(3), 44-59. https://doi.org/10.3390/admsci8030044

Hernández, J., Espinoza, J. y Aguilar, M. (2016). Diferencias en los motivadores y los valores en el trabajo de empleados en empresas maquiladoras. Contaduría y Administración, 61(1), 58-83. http://dx.doi.org/10.1016/j. cya.2015.09.003

Horstmanny, L. y Martín, J. (2017). Análisis del fenómeno del Employer Branding como herramienta de gestión de la atracción y retención del talento en las empresas. Revista Icade. Revista de las Facultades de Derecho y Ciencias Económicas y Empresariales, (100). https://doi.org/10.14422/icade.i100.y2017.008

Hwang, S. (2012). The mediating effect of emotional dissonance on the relationship between internal marketing and customer orientation: focused on the nursing organization. J Korea Serv Manag Soc, 13(2), 25-45. https:// doi.org/10.15706/jksms.2012.13.2.002

Jayasinghe, S. (2017). Effect of Employer Brand Image on Application Intentions of Final Year Management Undergraduates: Unfolding Employer Branding Determinants. Human Resource Management Journal, 5(1). 3548. https://doi.org/10.31357/hrmj.v5i1.3574

Jiménez, A. y Guardia, C. (2017). Revisión del concepto de internal branding: la transmisión de la promesa de marca a los clientes por parte de los empleados. Gráfica, 5(9), 29-37. https://doi.org/10.5565/rev/grafica.68

Kotler, P., y Keller, K. (2012). Dirección de marketing. México: D.F. Pearson educación. https://doi. org/10.1556/9789630597784

López Rodríguez, C. E., Acosta Molina, A., Páez León, A., Sarmiento Páez, L.M., y Tafur Serrano, A. (2018). Enfoques estratégicos del brand equity en las empresas que internacionalizan servicios de operación logística en la ciudad de Bogotá. Universidad \& Empresa, 20(35), 117-142. http://dx.doi.org/10.12804/revistas.urosario. edu.co/empresa/a.5570

Moroko, L. \& Uncles, M. D. (2009). Employer branding and market segmentation. Journal of Brand Management, 17(3), 181-196. https://doi.org/10.1057/bm.2009.10 
Narváez-Pereda, C. (2018). Importancia de la Admistración de Procesos de Negocio para el Área de Transportes de los Operadores Logísticos. Industrial Data, 21(1), 113-122. https://doi.org/10.15381/idata.v21i1.14926

Neirotti, P. \& Pesce, D. (2019). ICT-based innovation and its competitive outcome: The role of information intensity. European Journal of Innovation Management, 22(2), 383-404. https://doi.org/10.1108/EJIM-02-2018-0039 Olivos, P., Carrasco, F., Flores, J., Moreno, Y. y Nava, G. (2015). Modelo de gestión logística para pequeñas y medianas empresas en México. Contaduría y administración, 60(1), 181-203. https://doi.org/10.1016/S01861042(15)72151-0

Orjuela, J., Ocampo, Ó. y Bulla, E. (2005). Operadores y plataformas logísticas. Tecnura, 8(16), 115-127. https:// doi.org/10.14483/22487638.6249

Ortegón-Cortázar, L., y Royo-Vela, M. (2017). Attraction factors of shopping centers: Effects of design and eco-natural environment on intention to visit. European Journal of Management and Business Economics, 26(2), 199219. https://doi.org/10.1108/ejmbe-07-2017-012

Páramo-Morales, D. (2015). La teoría fundamentada (Grounded Theory), metodología cualitativa de investigación científica. Pensamiento \& gestión, (39), 1-7. https://doi.org/10.14482/pege.39.8439

Rani, K., \& Kumar, P. (2011). Employer Branding in IT Sector. Indian Journal of Applied Research, 3(9), $292-295$. https://doi.org/10.15373/2249555x/sept2013/86

Robledo, J., Arán, M. y Pérez-Aranda, J. (2015). Gestión del conocimiento y orientación al marketing interno en el desarrollo de ventajas competitivas en el sector hotelero. Investigaciones Europeas de Dirección y Economía de la Empresa, 21(2), 84-92. https://doi.org/10.1016/j.iedee.2014.03.001

Sánchez-Sellero, M., Sánchez-Sellero, P., Cruz-González, M. M., \& Sánchez-Sellero, F. (2018). Relación entre sobrecualificación y satisfacción laboral durante la crisis española de 2008. Contaduría y administración, 63(2), 1-20. http://dx.doi.org/10.22201/fca.24488410e.2018.1273

Sempértegui, C. \& Bravo, D. (2018). Endomarketing strategies in the coffee shops industry. PODIUM, (34), 21-34. https://doi.org/10.31095/podium.2018.34.2

Theurer, C. P., Tumasjan, A., Welpe, I. M., \& Lievens, F. (2018). Employer branding: a brand equity-based literature review and research agenda. International Journal of Management Reviews, 20(1), 155-179. https://doi. org/10.1111/ijmr.12121

Valencia, L. y Bernal, G. (2017). Diseño de un modelo de mejora para un operador logístico: análisis y evaluación. Caso "Distribuciones Delta SAS". Revista Sennova: Revista del Sistema de Ciencia, Tecnología e Innovación, 2(2), 232-260. https://doi.org/10.23850/23899573.558

Vega, J. y Serna, M. (2017). El marketing interno y la orientación al aprendizaje como antecedentes a la innovación en las pequeñas y medianas empresas en Aguascalientes. Revista Iberoamericana de Contaduría, Economía y Administración: RICEA, 6(11), 1-24. https://doi.org/10.23913/ricea.v6i11.86

Villegas, E., Hernández, M. y Salazar, B. (2017). La medición del capital intelectual y su impacto en el rendimiento financiero en empresas del sector industrial en México. Contaduría y Administración, 62(1), 184-206. http:// dx.doi.org/10.1016/j.cya.2016.10.002 


\section{Anexo}

\section{Instrumento para la recolección de la información}

Dinámicas del employer branding en las organizaciones que internacionalizan servicios de operación logística en Bogotá, Colombia

Un cordial saludo. Esta encuesta se realiza para obtener la información primaria de la investigación denominada "Dinámicas del employer branding en las organizaciones que internacionalizan servicios de operación logística en Bogotá, Colombia" liderada por los investigadores Campo Elías López-Rodríguez y Samir Ricardo Neme-Chaves. Su participación es voluntaria y la información suministrada por Ud. será completamente confidencial siendo esta utilizada únicamente para fines estadísticos y académicos. No existen respuestas correctas o incorrec- tas. Las preguntas relacionadas a continuación deben ser contestadas con una sola respuesta:

\section{1. ¿Cuál es su rango de edad?}
a. De 18 a 25 años
b. De 26 a 30 años
c. De 31 a 35 años
d. De 36 a 40 años
e. De 41 a 45 años
f. De 46 a 50 años
g. Más de 51 años

3. ¿A qué género pertenece?
a. Masculino
b. Femenino
c. Otro

2. ¿Cuál es su formación académica?
a. Bachiller
b. Técnico
c. Tecnólogo
d. Profesional
e. Especialista
f. Magíster
g. Doctor

4. ¿Cuál es su estado civil?
a. Soltero
b. Casado
c. Unión libre
d. Divorciado
e. Viudo 
5. ¿Cuál es o fue su condición laboral en la empresa?
a. Contrato a término fijo
b. Contrato a término indefinido
c. Orden de prestación de servicios
d. Contrato laboral por horas

6. ¿Cuánto tiempo está o estuvo vinculado laboralmente con la empresa?
a. Menos de un año
b. Entre uno y dos años
c. Entre tres y cuatro años
d. Entre cinco y seis años
e. Siete años o más

7. ¿A qué nivel jerárquico pertenece o perteneció dentro de la empresa?
a. Alta gerencia
b. Media gerencia
c. Nivel operativo

A continuación, se presenta una serie de frases relativamente cortas que permite hacer una descripción de cómo percibe a la organización en la que trabaja. Para ello debe responder con la mayor sinceridad posible a cada una de las oraciones que aparecen a continuación, de acuerdo a como piense o actúe, considerando la siguiente escala: $1=$ Totalmente en desacuerdo; $2=$ En desacuerdo; 3 = Ni de acuerdo, ni en desacuerdo; 4= De acuerdo; 5= Totalmente de acuerdo.

\begin{tabular}{|c|c|c|c|c|c|c|}
\hline \multicolumn{7}{|c|}{ Desarrollo } \\
\hline 8 & La empresa me capacita para mejorar los procesos que desarrollo dentro de ella. & 1 & 2 & 3 & 4 & 5 \\
\hline 9 & Recibo información respecto a la exigencia de los clientes de la empresa & 1 & 2 & 3 & 4 & 5 \\
\hline 10 & Sé que los clientes esperan que los servicios de la empresa sean de alta calidad & 1 & 2 & 3 & 4 & 5 \\
\hline 11 & La empresa me ofrece oportunidades para aumentar mi conocimiento a su favor. & 1 & 2 & 3 & 4 & 5 \\
\hline \multicolumn{7}{|c|}{ Contratación y retención } \\
\hline 12 & El proceso de reclutamiento de nuevos colaboradores es claro y adecuado. & 1 & 2 & 3 & 4 & 5 \\
\hline 13 & Las actividades y responsabilidades de los nuevos colaboradores son claramente definidas. & 1 & 2 & 3 & 4 & 5 \\
\hline 14 & Soy remunerado de forma justa de acuerdo con mis competencias y formación. & 1 & 2 & 3 & 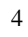 & 5 \\
\hline 15 & La empresa ofrece la oportunidad de recibir ingresos extras. & 1 & 2 & 3 & 4 & 5 \\
\hline 16 & Existen en la empresa reconocimientos a los colaboradores por sus logros. & 1 & 2 & 3 & 4 & 5 \\
\hline
\end{tabular}




\begin{tabular}{|c|c|c|c|c|c|c|}
\hline \multicolumn{7}{|c|}{ Adecuación al trabajo } \\
\hline 17 & Si el colaborador desea, él puede solicitar cambio de función y de área de gestión. & 1 & 2 & 3 & 4 & 5 \\
\hline 18 & $\begin{array}{l}\text { La empresa se preocupa por atribuir funciones de acuerdo con las habilidades y compe- } \\
\text { tencias de cada colaborador. }\end{array}$ & 1 & 2 & 3 & 4 & 5 \\
\hline 19 & $\begin{array}{l}\text { Tengo libertad y autonomía para tomar decisiones relativas en relación con mis activida- } \\
\text { des laborales. }\end{array}$ & 1 & 2 & 3 & 4 & 5 \\
\hline 20 & $\begin{array}{l}\text { En la empresa existen diferentes programas para atender las necesidades de los colabo- } \\
\text { radores. }\end{array}$ & 1 & 2 & 3 & 4 & 5 \\
\hline \multicolumn{7}{|c|}{ Comunicación interna } \\
\hline 21 & Las metas y los objetivos de la empresa son divulgados a todos los colaboradores. & 1 & 2 & 3 & 4 & 5 \\
\hline 22 & Conozco los resultados del área de trabajo en la cual me desempeño. & 1 & 2 & 3 & 4 & 5 \\
\hline 23 & $\begin{array}{l}\text { Tengo la oportunidad de expresar mis puntos de vista en relación con las actividades del } \\
\text { área de trabajo en la cual me desempeño. }\end{array}$ & 1 & 2 & 3 & 4 & 5 \\
\hline 24 & Conozco los resultados generales de la empresa. & 1 & 2 & 3 & 4 & 5 \\
\hline 25 & $\begin{array}{l}\text { Los cambios que van a ocurrir a nivel general en la empresa son comunicados con antici- } \\
\text { pación a todos los colaboradores. }\end{array}$ & 1 & 2 & 3 & 4 & 5 \\
\hline 26 & $\begin{array}{l}\text { Los cambios que van a ocurrir específicamente en el área de trabajo en la cual me desem- } \\
\text { peño son comunicados con anticipación a todos los colaboradores. }\end{array}$ & 1 & 2 & 3 & 4 & 5 \\
\hline 27 & Conozco claramente la misión, visión, objetivos, políticas y valores de la empresa. & 1 & 2 & 3 & 4 & 5 \\
\hline \multicolumn{7}{|c|}{ Comunicación externa } \\
\hline 28 & Las oportunidades que llegan a la empresa son comunicadas a los colaboradores. & 1 & 2 & 3 & 4 & 5 \\
\hline 29 & Las amenazas que llegan a la empresa son comunicadas a los colaboradores. & 1 & 2 & 3 & 4 & 5 \\
\hline 30 & $\begin{array}{l}\text { El lanzamiento de nuevos productos es comunicado inicialmente en la empresa y sólo } \\
\text { después a los clientes. }\end{array}$ & 1 & 2 & 3 & 4 & 5 \\
\hline 31 & La empresa hace partícipe a los colaboradores en las estrategias de comunicación. & 1 & 2 & 3 & 4 & 5 \\
\hline
\end{tabular}

\section{Consentimiento informado}

Este documento tiene como fin recibir el consentimiento para usar la información resultado de las encuestas, para ampliar la información sobre la gestión employer branding en las organizaciones que internacionalizan servicios de operación logística en la ciudad de Bogotá, Colombia. La muestra estadísticamente representativa para la investigación es de 384 encuestas (nivel de confianza del 95\%, margen de error del 5\% y variabilidad positiva y negativa del $50 \%$ ) dirigidas a los clientes internos de las organizaciones que internacionalizan servicios de operación logística en la ciudad de Bogotá Colombia. Es importante conocer la percepción de ustedes en la información que brindan, ya que contribuirá para la construcción de información para el sector y la industria de la capital colombiana y dar cumplimiento al objetivo planteado en la investigación. El instrumento utilizado es una encuesta que cuenta con preguntas de selección múltiple con una única respuesta y de escala Likert; instrumento que se tabulará y generará tendencias de información para un análisis de comportamientos frente a las varia- 
bles estudiadas. Su valiosa aceptación para el uso de la información es decisión de cada uno de ustedes y será aprobada mediante la conformidad de este documento. En caso de querer obtener los resultados de la encuesta por favor contactar al investigador Campo Elías López Rodríguez clopezr3@uniminuto.edu.co

Para el consentimiento usted diligenciará y aceptará que:

- He sido informado de los objetivos de la investigación.

- Corresponde a la sección en donde se firma el Consentimiento.

- Incluye información clara y precisa de la investigación, relativa al propósito del estudio, modalidad de participación, beneficios, voluntariedad, derecho a conocer los resultados, derecho a retirarse del estudio en cualquier momento, voluntariedad, confidencialidad, información del IR, del Patrocinante y del Comité Ético Científico.

ACEPTO Sí No 